\title{
MATHEMATICAL PERSPECTIVES
}

BULLETIN (New Series) OF THE

AMERICAN MATHEMATICAL SOCIETY

Volume 47, Number 4, October 2010, Pages 695-696

S 0273-0979(2010)01316-1

Article electronically published on July 27, 2010

\section{ABOUT THE COVER: THE MATHEMATICAL IMAGERY OF LUN-YI TSAI}

\author{
ARAVIND ASOK
}

For a (real) parameter $t$ and coordinates $(x, y, z)$ on $\mathbb{R}^{3}$, consider the family of quadric surfaces defined by the equations

$$
-x^{2}-y^{2}+z^{2}=t
$$

As $t$ varies, visualize the graph of the resulting equation; these graphs arise in a number of unrelated situations. In multivariable calculus we discuss the graphs of hypersurfaces in this family with our students. Even though general relativity considers 4-dimensional Lorentzian manifolds, the cone obtained by setting $t=0$ in the above equation provides a standard picture for the light cone or null cone (cf. 11, Figure 2.1]). Equation 1 continues to makes sense if we replace the field of real numbers by an arbitrary field $k$; this is the domain of algebraic geometry. If $k$ has characteristic unequal to 2 , this family provides an example of a degeneration of a nonsingular variety to a singular variety. Even when working with varieties over an arbitrary field, many algebraic geometers find it useful to keep the "real" picture in mind.

The cover and the following picture display American Degeneration, a painting by artist Lun-Yi Tsai. A mathematician looking at these images might be reminded of Morse theory, general relativity, or the algebro-geometric theory of singularities. On the other hand, a nonmathematician looking at the same image might have different reactions. In full color, it is hard not to be reminded of a flag. Indeed, this particular image is part of a series painted while the artist was working in Berlin and, coincidentally, during Germany's Jahr der Mathematik (2008). The colors of this painting are those of the American flag and another in the series (European Dream) is composed of the colors of the German flag [3. Tsai says these paintings are an examination of his "identity as an American artist in that very creative German capital." In black and white, perhaps viewed in landscape, one might see

(C)2010 American Mathematical Society Reverts to public domain 28 years from publication 
an aerial view of a Richard Serra sculpture. This juxtaposition of mathematical imagery with "everyday" objects is one aspect of Tsai's art.

Lun-Yi Tsai is a mathematical artist: Tsai grew up among artists in New York (this particular group of artists has been profiled in, e.g., [2]), and was also formally trained in mathematics (he received an M.A. in mathematics from the University of Pittsburgh). He currently teaches mathematics and makes art in Miami, Florida. During his residence in Germany he was selected as "Mathmaker of the week" for building bridges between mathematics and art. In addition to his personal explorations of the relationship between mathematics and art, a number of his recent pieces involved direct collaboration between Tsai and a mathematician.

Among other things, Tsai's artwork questions the perception of mathematical images. On the one hand, he is interested in how mathematical concepts appear in every day life. On the other hand, Tsai attempts to convey to nonmathematicians those aspects of mathematics that mathematicians themselves find beautiful, e.g., conceptual simplicity in the structure of a proof, or mutual interconnectedness of $a$ priori diverse ideas. Tsai's version of mathematical art avoids mere appropriation of mathematical symbols and graphics, or the "engineering" of works by means of computers or 3D printers.

One of Tsai's collections (Demonstrations) consists of charcoal drawings superimposed upon related mathematical texts, e.g., handwritten redaction of notes from lectures, transcripts of conversations, or material from textbooks. Some of these drawings bring to mind the marginal notes and pictures one makes while reading a book or paper [3]. Other pieces present simple pictures that communicate geometric proofs (recall the pictorial proof of the Pythagorean theorem using two inscribed squares of different side lengths). All of Tsai's work attempts to convey in a visually appealing and nontechnical fashion the joy mathematicians derive from the act of doing mathematics.

\section{REFERENCES}

[1] P. Chrusciel, G. Galloway, and D. Pollack, Mathematical general relativity: A sampler, Bull. Amer. Math. Soc. 47 (2010), 567-638.

[2] R. Kostelanetz, SoHo: The Rise and Fall of an Artists' Colony, Routledge, 2003.

[3] L.-Y. Tsai, webpage: www.lunyitsai.com, 1, 2.

Department of Mathematics, University of Southern California, 3620 S. Vermont Ave KAP 104, Los Angeles, California 90089

E-mail address: asok@usc.edu 